\title{
The environs of the ultracompact HII region G45.45+0.06
}

\author{
S. Paron, S. Cichowolski, and M. E. Ortega \\ Instituto de Astronomía y Física del Espacio (IAFE), CC 67, Suc. 28, 1428 Buenos Aires, Argentina \\ e-mail: sparon@iafe.uba.ar
}

Received 5 June 2009 / Accepted 27 July 2009

\begin{abstract}
Aims. G45.45+0.06 is an ultra-compact HII (UCHII) region that has been extensively studied. It is known that G45.45+0.06 is embedded in a complex of UCHII regions, but to date, the surrounding ISM on a larger spatial scale has not been analyzed. Methods. Using data from large-scale surveys, the Two Micron All Sky Survey, GLIMPSE, MIPSGAL, MAGPIS and GRS, we performed a multiwavelength study of a region of about $7^{\prime} \times 7^{\prime}$ in the vicinity of G45.45+0.06.

Results. We found that the UCHII complex lies on the border of a larger (diameter of $\sim 3^{\prime}$ ) and fainter HII region, which is located at the same distance as G45.45+0.06, 8 kpc. In this work, this larger HII region is called G45L. A good morphological correlation is observed between the PDRs and the molecular gas mapped in the ${ }^{13} \mathrm{CO} J=1-0$ and $\mathrm{CS} J=2-1$ lines, suggesting that G45L may be collecting molecular material. From a near- and mid-IR photometric study, we found three sources, likely O-type stars, that are possibly responsible for the creation of G45L. Additionally, we found several YSO candidates lying preferentially in the molecular shell that surrounds G45L. Our results confirm that the region southeast of the UCHII complex where G45.45+0.06 is embedded and east of G45L is undergoing active star formation. We suggest that G45L has been expanding for about $2 \times 10^{6}$ yr and could have triggered the formation of the zero-age main sequence stars that ionize the UCHII region G45.45+0.06. However we cannot discard that both HII regions are coeval.
\end{abstract}

Key words. ISM: HII regions - ISM: clouds - stars: formation

\section{Introduction}

It is well established that the formation of massive stars can be triggered by the action of expanding HII regions through the "collect and collapse" process. During its supersonic expansion, an HII region can collect a dense layer of material between its ionization and shock fronts. This layer can be fragmented in massive condensations that then may collapse to lead to the formation of new stars. Thus, it is possible to find protostars, young stars, and ultra-compact HII (UCHII) regions on the borders of HII regions. Several pieces of observational evidence have been found supporting this star formation mechanism (see e.g. Pomarès et al. 2009; Zavagno et al. 2007, and reference therein).

G45.45+0.06 is a luminous Galactic UCHII region that has been extensively studied in the radio continuum and in molecular lines (e.g. Wood et al. 1988; Garay et al. 1993; Wilner et al. 1996; Hatchell et al. 1998). This UCHII region presents $\mathrm{CH}_{3} \mathrm{OH}$ and $\mathrm{H}_{2} \mathrm{O}$ maser emission (Codella et al. 2004). Recently, G45.45+0.06 was included in the Boston University Catalog of Galactic HII Region Properties (Anderson et al. 2009). Using the ${ }^{13} \mathrm{CO} J=1-0$ emission obtained from the GRS ${ }^{1}$, the authors derived a $v_{\mathrm{LSR}} \sim 55.6 \mathrm{~km} \mathrm{~s}^{-1}$ and a kinematical distance of $8.2 \mathrm{kpc}$ for this UCHII region, in agreement, within errors, with previous estimations (e.g. $v_{\mathrm{LSR}} \sim 55.9 \mathrm{~km} \mathrm{~s}^{-1}$ and $d \sim$ $7.7 \mathrm{kpc}$, Kolpak et al. 2003). As proposed by Matthews et al. (1977), G45.45+0.06 is part of a cluster of several UCHII regions. Giveon et al. (2005a,b) generated a catalog matching VLA Galactic plane catalogs at 5 and $1.4 \mathrm{GHz}$ with new radio continuum observations and the MSX6C Galactic plane catalog. According to this catalog, G45.45+0.06 is part of a complex of five radio compact HII regions. Feldt et al. (1998) performed a near- and mid-infrared study of G45.45+0.06 and concluded

${ }^{1}$ Galactic Ring Survey (Jackson et al. 2006). that this UCHII region, the oldest in the complex, is a young OB cluster around which sequential star formation is taking place. Recently, Blum \& McGregor (2008) using NIFS behind ALTAIR on Gemini North identified several massive O-type stars that are ionizing G45.45+0.06. The complexity of this region is evident. Moreover, in the vicinity of this complex (see Fig. 1), Cyganowski et al. (2008) discovered an "extended green object" (EGO), a source with extended Spitzer-IRAC $4.5 \mu \mathrm{m}$ emission which is usually presented in green colour. According to the authors, an EGO is a massive young stellar object (MYSO) driven outflow. The presence of this EGO reveals that star formation is taking place in this region. Indeed, this EGO coincides with the high-mass star forming region G45.47+0.05 studied by Remijan et al. (2004).

In this work, we present a multiwavelength study of the spatial environment surrounding the UCHII region G45.45+0.06, with the purpose of exploring the ISM around the complex. We use survey and archival data to show that G45.45+0.06 is located in the environs of a larger HII region whose expansion could have generated the conditions for the formation of the UCHII region G45.45+0.06.

\section{Data}

The data presented and analyzed in this work were extracted from five large-scale surveys: the Two Micron All Sky Survey (2MASS) ${ }^{2}$, Galactic Legacy Infrared Mid-Plane Survey Extraordinaire (GLIMPSE), MIPSGAL, MAGPIS and GRS.

\footnotetext{
2 2MASS is a joint project of the University of Massachusetts and the Infrared Processing and Analysis Center/California Institute of Technology, funded by the National Aeronautics and Space Administration and the National Science Foundation.
} 
GLIMPSE is a mid infrared survey of the inner Galaxy performed using the Spitzer Space Telescope. We used the mosaiced images from GLIMPSE and the GLIMPSE Point-Source Catalog (GPSC) in the Spitzer-IRAC (3.6, 4.5, 5.8 and $8 \mu \mathrm{m})$. IRAC has an angular resolution of between $1^{\prime \prime} .5$ and 1.9 (see Fazio et al. 2004; Werner et al. 2004). MIPSGAL is a survey of the same region as GLIMPSE, using the MIPS instrument (24 and $70 \mu \mathrm{m}$ ) on Spitzer. The MIPSGAL resolution at $24 \mu \mathrm{m}$ is $6^{\prime \prime}$. MAGPIS is a radio continuum survey of the Galactic plane at 6 and $20 \mathrm{~cm}$ using the VLA in configurations B-D combined with the Effelsberg $100 \mathrm{~m}$ single-dish telescope (White et al. 2005). The GRS is being carried out by the Boston University and the Five College Radio Astronomy Observatory (FCRAO). The survey maps the Galactic Ring in the ${ }^{13} \mathrm{CO} J=1-0$ line with an angular and spectral resolution of $46^{\prime \prime}$ and $0.2 \mathrm{~km} \mathrm{~s}^{-1}$, respectively (see Jackson et al. 2006). The observations were performed in both position-switching and On-The-Fly mapping modes, achieving an angular sampling of $22^{\prime \prime}$. We also analyzed the additional data that this survey produces: the CS $J=$ 2-1 line, which has similar angular and spectral resolution as the ${ }^{13} \mathrm{CO} J=1-0$ line.

\section{Results and discussion}

Figure 1 shows a Spitzer-IRAC three color image extracted from GLIMPSE, of a region about $7^{\prime} \times 7^{\prime}$ in the vicinity of the UCHII complex where G45.45+0.06 lies. The three IR bands are $3.6 \mu \mathrm{m}$ (in blue), $4.5 \mu \mathrm{m}$ (in green) and $8 \mu \mathrm{m}$ (in red). The contours correspond to the ${ }^{13} \mathrm{CO} J=1-0$ emission as extracted from the GRS integrated between 50 and $65 \mathrm{~km} \mathrm{~s}^{-1}$, a velocity range around the $v_{\mathrm{LSR}}$ of G45.45+0.06. The UCHII complex lies on a border of a more extended structure as seen mainly in the Spitzer-IRAC $8 \mu \mathrm{m}$ band. The morphological correlation between the $8 \mu \mathrm{m}$ band, mainly originating from polycyclic aromatic hydrocarbons (PAHs), and the molecular gas is suggestive of an expanding HII region. The PAH emission delineates the HII region boundaries. This is because these molecules are destroyed inside the ionized region, but are excited in the photodissociation region (PDR) by the radiation leaking from the HII region (Pomarès et al. 2009).

Although G45L is not completely bordered by a PDR, its morphology resembles the structure of the IR dust bubbles associated with $\mathrm{O}$ and early-B type stars: a PDR visible in the $8 \mu \mathrm{m}$ band, which encloses ionized gas observed at $20 \mathrm{~cm}$ and hot dust observed at $24 \mu \mathrm{m}$ (see Churchwell et al. 2006, 2007; Watson et al. 2008). Figure 2 (left) displays a composite two color image, where the red represents the Spitzer-IRAC $8 \mu \mathrm{m}$ band and the green the above $3 \sigma$ radio continuum emission at $20 \mathrm{~cm}$ extracted from MAGPIS. The high angular resolution of the MAGPIS $20 \mathrm{~cm}$ image allows us to distinguish two different radio continuum structures: that associated with the UCHII complex, where both colors are combined and satured (yellow), and other, more diffuse, towards the west, enclosed by the $8 \mu \mathrm{m}$ emission that indicates the photodissociation regions (PDRs). Hereafter, this latter structure, an HII region of about $3^{\prime}$ in diameter, possibly related to the molecular gas shown in Fig. 1 as contours, will be called G45L. As indicated in Fig. 2 (left), the brightest part of the radio continuum diffuse structure located at $l \sim 45.42, b \sim 0.072$ is bordered by two PDRs: the north and the middle PDR. Towards the south, the HII region is fainter and bordered by what we called south PDR. Figure 2 (right) shows a two color image where again the red is the Spitzer-IRAC $8 \mu \mathrm{m}$ emission and the blue represents the MIPSGAL emission at $24 \mu \mathrm{m}$. The red structure observed in the UCHII complex

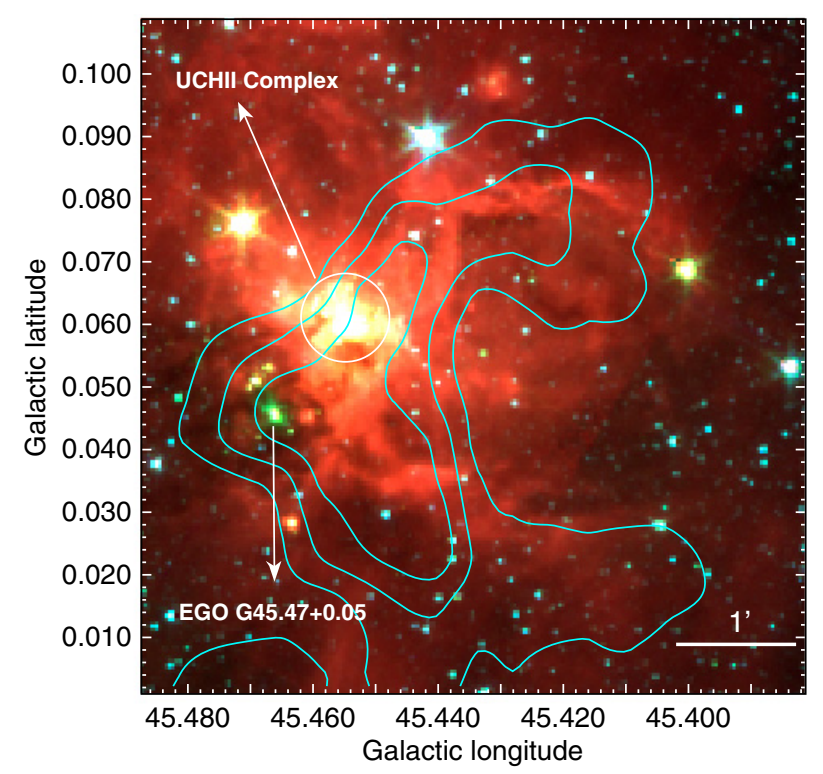

Fig. 1. Spitzer-IRAC three color image $(3.5 \mu \mathrm{m}=$ blue, $4.5 \mu \mathrm{m}=$ green and $8 \mu \mathrm{m}=$ red). The contours correspond to the ${ }^{13} \mathrm{CO} J=1-0$ emission as extracted from the GRS integrated between 50 and $65 \mathrm{~km} \mathrm{~s}^{-1}$, the levels are 27, 37 and $48 \mathrm{~K} \mathrm{~km} \mathrm{~s}^{-1}$. The UCHII complex is remarked with a circle. Also, the EGO discovered by Cyganowski et al. (2008) is indicated. The angular resolutions are $\sim 1$.' 5 and $\sim 46^{\prime \prime}$ for the Spitzer and molecular data, respectively.

is not real, it is due to the presence of saturated pixels in the MIPSGAL image.

\subsection{Distance}

We are studying a region in the first Galactic quadrant thus, we have to take into account the distance ambiguity that exists when using radial velocities and a Galactic rotation curve to assign distances to sources. According to Kolpak et al. (2003) and Anderson et al. (2009), G45.45+0.06 has a radio recombination line velocity of $\sim 56 \mathrm{~km} \mathrm{~s}^{-1}$, which gives possible distances of $\sim 4$ or $\sim 8 \mathrm{kpc}$. As the $\mathrm{HI}$ absorption spectrum towards G45.45+0.06 presents an absorption feature at the tangent point velocity $\left(\sim 64 \mathrm{~km} \mathrm{~s}^{-1}\right)$, the authors adopted the farther distance for $\mathrm{G} 45.45+0.06$.

The molecular gas belonging to the shell associated with G45L (see Fig. 1) is in the same velocity range as the gas related to the UCHII complex where G45.45+0.06 is embedded, which may suggest that $\mathrm{G} 45 \mathrm{~L}$ is at the same distance as the UCHII complex. In order to prove this suggestion, using HI data extracted from the VLA Galactic Plane Survey (VGPS) (Stil et al. 2006), we studied the absorption features towards two regions: the UCHII complex where G45.45+0.06 is embedded (Fig. 3-up) and the radio continuum structure enclosed by the north and middle PDR (Fig. 3-down). As both profiles have the same HI absorption features, we conclude that G45L is at the same distance as G45.45+0.06. Hereafter, we adopt the distance of $8 \mathrm{kpc}$.

\subsection{Molecular environment}

As shown in Fig. 1, an incomplete ${ }^{13} \mathrm{CO}$ shell at the velocity range between 50 and $65 \mathrm{~km} \mathrm{~s}^{-1}$ presents a good morphological correlation with the north and south PDRs visible in the $8 \mu \mathrm{m}$ band (see Fig. 2-left). This suggests that the expansion 

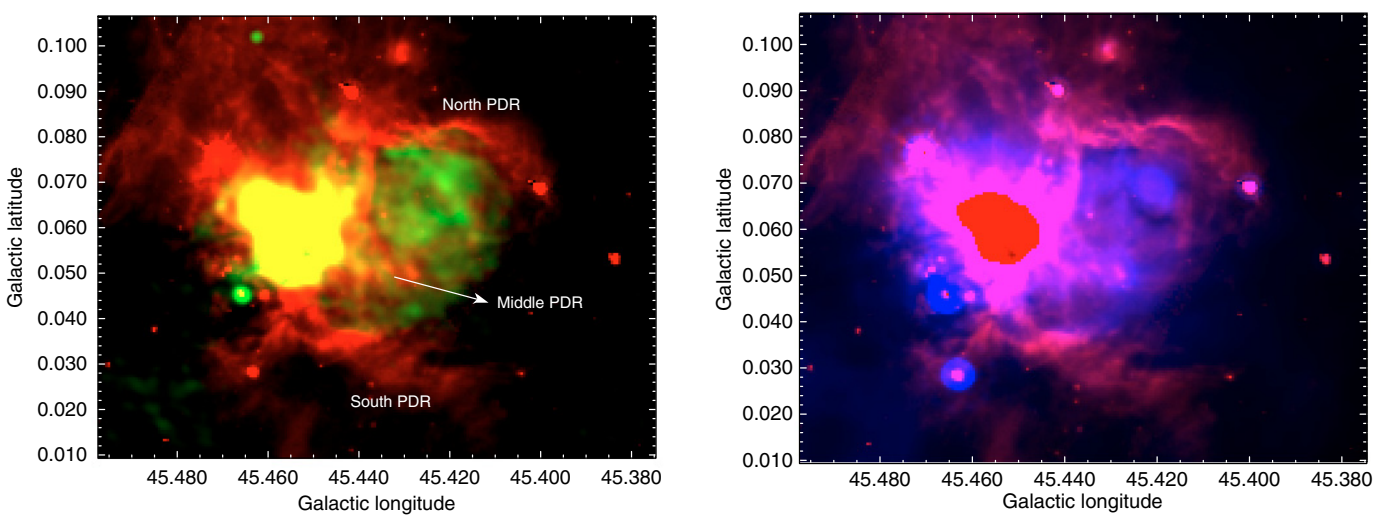

Fig. 2. Two color images. Left: the red is the Spitzer-IRAC $8 \mu \mathrm{m}$ emission and the green is the above $3 \sigma$ radio continuum emission at $20 \mathrm{~cm}$ extracted from MAGPIS. Yellow is the superposition of the IR and radio continuum emissions. Right: the red is the same as the left image and the blue represents the MIPSGAL emission at $24 \mu \mathrm{m}$. The red structure onto the UCHII complex is not real, it is due to the presence of saturated pixels of the MIPSGAL emission.
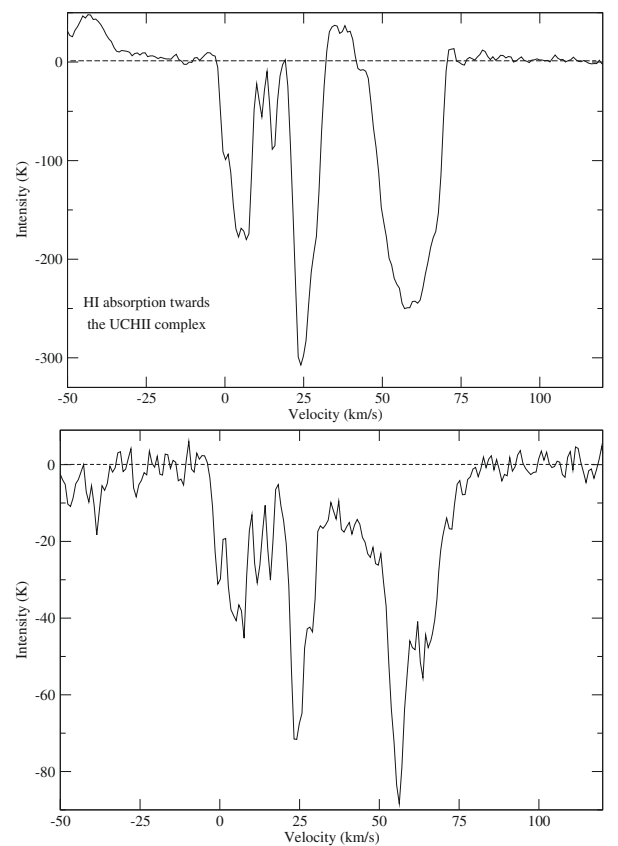

Fig. 3. Up: HI absorption towards the UCHII complex where G45.45+0.06 is embedded. Down: HI absorption towards the radio continuum structure enclosed by the north and middle PDR. Note that both profiles have the same HI absorption features.

of the HII region, that we called G45L, is collecting the molecular gas, as is observed in other HII regions (e.g. Pomarès et al. 2009; Cichowolski et al. 2009; Deharveng et al. 2008). Figure 4 shows the integrated velocity channel maps of the ${ }^{13} \mathrm{CO} J=$ $1-0$ emission every $\sim 1.25 \mathrm{~km} \mathrm{~s}^{-1}$. For reference, the circle highlights the position and the size of G45L as suggested by the north and south PDRs. From this figure is clear that the molecular gas is more abundant towards the east. In particular, the $v=58.1 \mathrm{~km} \mathrm{~s}^{-1}$ channel map shows two molecular clumps spatially coincident with the UCHII complex and the region where the EGO G45.47+0.05 lies, respectively. It is also evident that the molecular gas encompasses the circle, showing the velocity structure of the molecular shell related to G45L. As Deharveng et al. (2005) propose, the presence of a molecular shell surrounding the ionized gas of an HII region, or the presence of massive fragments regularly spaced along the ionization front, suggest

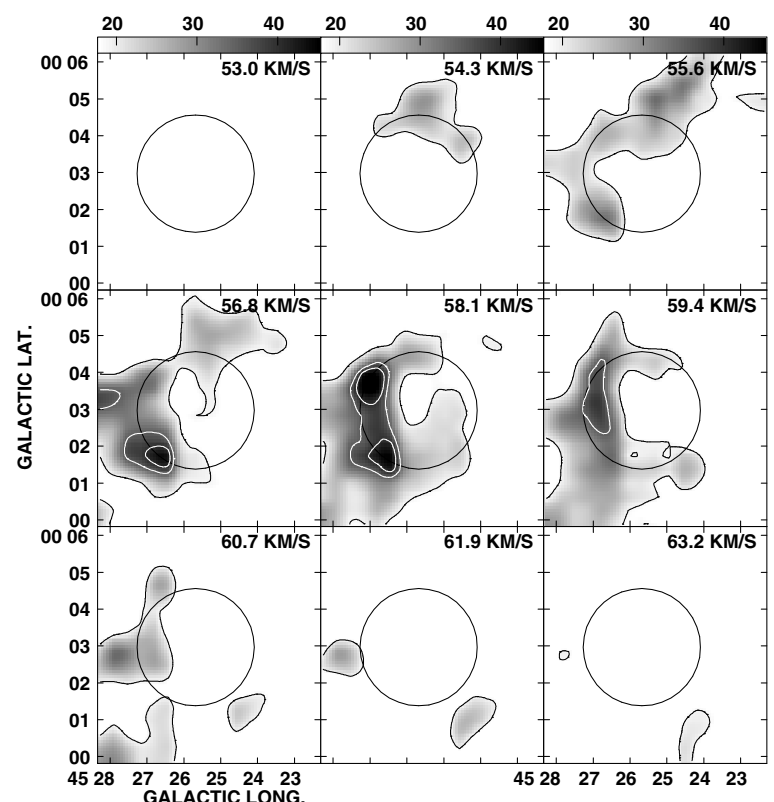

Fig. 4. Integrated velocity channel maps of the ${ }^{13} \mathrm{CO} J=1-0$ emission every $\sim 1.25 \mathrm{~km} \mathrm{~s}^{-1}$. The grayscale is displayed at the top of the figure and is in $\mathrm{K} \mathrm{km} \mathrm{s}^{-1}$, the contour levels are 5, 9.5 and $11 \mathrm{~K} \mathrm{~km} \mathrm{~s}^{-1}$. Note that the molecular gas encompasses the circle, which highlights the position and size of G45L as suggested by the north and south PDRs (see Fig. 2-left).

that the collect and collapse process is at work in the region. On the other hand, the channels with velocities of 56.8 and $58.1 \mathrm{~km} \mathrm{~s}^{-1}$ show a smaller molecular shell interior to the circle and in spatial coincidence with the structure delimited by the middle and north PDRs (see Fig. 2-left).

Figure 5 displays the CS $J=2-1$ emission integrated between 54 and $66 \mathrm{~km} \mathrm{~s}^{-1}$. The CS $J=2-1$ emission presents a similar structure to the ${ }^{13} \mathrm{CO}$. Given that this line requires high densities, $10^{4}-10^{5} \mathrm{~cm}^{-3}$, to be excited (see e.g. Luna et al. 2006), its detection indicates the presence of high density gas in the molecular shell, mainly towards the east.

In order to analyze the kinematics of the molecular gas, we study the molecular spectra from three different regions: Region 1 (the north portion of the molecular shell, in 


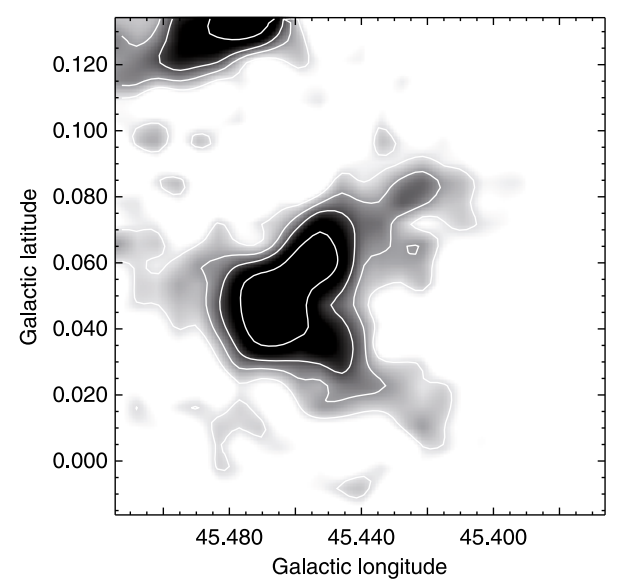

Fig. 5. CS $J=2-1$ emission integrated between 54 and $66 \mathrm{~km} \mathrm{~s}^{-1}$. The contour levels are 2.4, 3.6, 5 and $8 \mathrm{~K} \mathrm{~km} \mathrm{~s}^{-1}$. Note that the CS emission presents a similar structure as the ${ }^{13} \mathrm{CO}$.

Table 1. Observed parameters of the ${ }^{13} \mathrm{CO} J=1-0$ and CS $J=2-1$ average emissions towards the regions shown in Fig. 6.

\begin{tabular}{ccccc}
\hline \hline Emission & $\begin{array}{c}T_{\mathrm{mb}} \\
(\mathrm{K})\end{array}$ & $\begin{array}{c}V_{\mathrm{lsr}} \\
\left(\mathrm{km} \mathrm{s}^{-1}\right)\end{array}$ & $\begin{array}{c}\Delta v \\
\left(\mathrm{~km} \mathrm{~s}^{-1}\right)\end{array}$ & $\begin{array}{c}I \\
\left(\mathrm{~K} \mathrm{~km} \mathrm{~s}^{-1}\right)\end{array}$ \\
\hline \multicolumn{5}{c}{ Region 1 } \\
${ }^{13} \mathrm{CO} J=1-0$ & $3.80 \pm 0.50$ & $58.75 \pm 1.05$ & $5.05 \pm 0.25$ & $17.00 \pm 3.00$ \\
& $4.10 \pm 0.50$ & $55.25 \pm 1.05$ & $3.00 \pm 0.15$ & $15.00 \pm 2.00$ \\
$\mathrm{CS} J=2-1$ & $0.46 \pm 0.11$ & $59.60 \pm 1.10$ & $4.00 \pm 0.20$ & $2.00 \pm 0.30$ \\
& $0.42 \pm 0.15$ & $55.80 \pm 1.10$ & $3.45 \pm 0.15$ & $1.90 \pm 0.30$ \\
\hline \multicolumn{5}{c}{ Region 2} \\
${ }^{13} \mathrm{CO} J=1-0$ & $4.10 \pm 0.50$ & $59.50 \pm 0.50$ & $6.75 \pm 0.85$ & $30.00 \pm 3.80$ \\
$\mathrm{CS} J=2-1$ & $0.35 \pm 0.05$ & $59.90 \pm 0.50$ & $6.25 \pm 0.95$ & $2.30 \pm 0.50$ \\
\hline \multicolumn{5}{c}{ Region 3 } \\
${ }^{13} \mathrm{CO} J=1-0$ & $6.40 \pm 0.50$ & $58.65 \pm 0.25$ & $6.10 \pm 0.50$ & $41.50 \pm 3.50$ \\
$\mathrm{CS} J=2-1$ & $1.00 \pm 0.10$ & $59.25 \pm 0.55$ & $7.00 \pm 1.00$ & $7.70 \pm 1.50$ \\
\hline \multicolumn{5}{c}{}
\end{tabular}

coincidence with the north PDR), Region 2 (the south portion of the molecular shell, in coincidence with the south PDR) and Region 3 (where the molecular emission peaks). Figure 6 (left) shows the Spitzer-IRAC $8 \mu \mathrm{m}$ emission with contours of the integrated ${ }^{13} \mathrm{CO} J=1-0$ as presented in Fig. 1. Regions $1-3$ are indicated with boxes of approximately $2^{\prime} \times 11^{\prime} .8,2$. $2 \times 1.2$ and $1.5 \times 2$ '.5 in size, respectively. Towards the right, the ${ }^{13} \mathrm{CO} J=$ $1-0$ and CS $J=2-1$ average spectra corresponding to the emission of each region are displayed. Between 40 and 50 spectra were averaged to obtain each average emission spectrum. The parameters determined from Gaussian fitting of these lines are presented in Table 1. $T_{\mathrm{mb}}$ represents the peak brightness temperature, $V_{\mathrm{lsr}}$ the central velocity, $\Delta v$ the line width and $I$ the integrated line intensity. Errors are formal $1 \sigma$ value for the model of the Gaussian line shape. The ${ }^{13} \mathrm{CO}$ and CS average lines corresponding to Region 1 were best fitted with two Gaussians.

Besides, taking into account that the ${ }^{13} \mathrm{CO} J=1-0$ beam (46") completely covers the middle PDR, we present in Fig. 7 a spectrum obtained from $l=45^{\circ} .430, b=0.055$. This spectrum shows the molecular emission probably related to the middle PDR. The velocity of the main component of this profile is $v \sim 56 \mathrm{~km} \mathrm{~s}^{-1}$, coinciding with that of one of the two molecular components of the north PDR (Region 1 in Fig. 6). The spectrum is not symmetrical: it presents another weaker component or a wing towards larger velocities.

The molecular spectra analysis shows that the north PDR has two possible molecular structures associated, centered at $v \sim$
$55 \mathrm{~km} \mathrm{~s}^{-1}$ and $v \sim 59 \mathrm{~km} \mathrm{~s}^{-1}$ respectively. The main velocity component of the molecular gas associated with the middle PDR is centered at $v \sim 55 \mathrm{~km} \mathrm{~s}^{-1}$, while the molecular gas related to the south PDR is centered at $v \sim 59 \mathrm{~km} \mathrm{~s}^{-1}$. If the molecular gas is indeed associated with $\mathrm{G} 45 \mathrm{~L}$, this may suggest that we have to take into account projection effects to understand the three dimensional structure of G45L. This will be discussed in Sect. 3.5.

Using the ${ }^{13} \mathrm{CO} J=1-0$ line and assuming local thermodynamic equilibrium (LTE) we estimate the $\mathrm{H}_{2}$ column density towards the three regions shown in Fig. 6. We use:

$N\left({ }^{13} \mathrm{CO}\right)=2.42 \times 10^{14} \frac{T_{\mathrm{ex}} \int \tau_{13} \mathrm{~d} v}{1-\exp \left(-5.29 / T_{\mathrm{ex}}\right)}$

to obtain the ${ }^{13} \mathrm{CO}$ column density. $\tau_{13}$ is the optical depth of the line and following Anderson et al. (2009), the $T_{\text {ex }}$ was assumed to be $13 \mathrm{~K}$. We assume that the ${ }^{13} \mathrm{CO}$ emission is optically thin and use the Gaussian fit line parameters (Table 1) to find the optical depth integral. Finally, we use the relation $N\left(\mathrm{H}_{2}\right) / N\left({ }^{13} \mathrm{CO}\right) \sim$ $5 \times 10^{5}$ (e.g. Simon et al. 2001) to estimate the following values: $N\left(\mathrm{H}_{2}\right) \sim 1.5 \times 10^{22} \mathrm{~cm}^{-2}$ for Region 1 and 2, respectively and $N\left(\mathrm{H}_{2}\right) \sim 2.8 \times 10^{22} \mathrm{~cm}^{-2}$ for Region 3 .

In addition we estimate the CS column densities, $N(\mathrm{CS})$, towards these regions. Although the CS $J=2-1$ line is generally optically thick, a lower limit to the $N(\mathrm{CS})$ can be estimated under the assumption of optically thin CS emission. We use the following equation (Ohashi et al. 1991):

$$
N(\mathrm{CS})=8.5 \times 10^{11} \frac{\exp \left(2.4 / T_{\mathrm{ex}}\right)}{1-\exp \left(-4.7 / T_{\mathrm{ex}}\right)} \times T_{\mathrm{mb}} \times \Delta v,
$$

where $T_{\mathrm{mb}}$ is the CS brightness temperature and $\Delta v$ is the velocity width of the CS line. Following Goicoechea et al. (2006), who studied the Horsehead PDR using CS $J=2-1$ between other molecular lines, we assume $T_{\mathrm{ex}}=9 \mathrm{~K}$. We obtain $N(\mathrm{CS}) \sim$ $(8$ and 6$) \times 10^{12} \mathrm{~cm}^{-2}$ for Region 1 and 2 , respectively, and $\mathrm{N}(\mathrm{CS}) \sim 2 \times 10^{13} \mathrm{~cm}^{-2}$ for Region 3 .

From the $\mathrm{H}_{2}$ and CS column densities, we can estimate the CS abundances, $X(\mathrm{CS})=N(\mathrm{CS}) / N\left(\mathrm{H}_{2}\right)$. We obtain $X(\mathrm{CS}) \sim(5$, 3.5 and 7$) \times 10^{-10}$ for Region $1-3$, respectively. Taking into account the LTE approximation and that the estimated CS column densities are lower limits, these values are comparable with those obtained towards the Orion Bar (a warm PDR), $X(\mathrm{CS}) \sim 2.9 \times$ $10^{-9}$ (Johnstone et al. 2003) and towards the Horsehead PDR, $X(\mathrm{CS}) \sim(7 \pm 3) \times 10^{-9}$ (Goicoechea et al. 2006). New CS and $\mathrm{C}^{34} \mathrm{~S}$ observations in several lines would be very useful to study the sulfur depletion in G45L PDRs.

Finally, we estimate the total mass of the whole molecular shell in $M \sim 10^{5} M_{\odot}$. This value was obtained from:

$M=\mu m_{\mathrm{H}} \sum\left[D^{2} \Omega N\left(\mathrm{H}_{2}\right)\right]$

where $\Omega$ is the solid angle subtended by the ${ }^{13} \mathrm{CO} J=1-0$ beam size, $m_{\mathrm{H}}$ is the hydrogen mass, $\mu$, the mean molecular weight, is assumed to be 2.8 by taking into account a relative helium abundance of $25 \%$, and $D$ is the distance. Summation was performed over all the observed positions within the $27 \mathrm{~K} \mathrm{~km} \mathrm{~s}^{-1}$ contour level (see Figs. 1 and 6).

\subsection{The exciting star(s) of $G 45 L$}

Given that we do not find any cataloged massive star in the area that may be related to G45L, we have used the GLIMPSE I Spring'07 Catalog to analyze the infrared sources seen in projection onto G45L to identify the star(s) responsible for its creation. Considering only the sources that have been detected in 


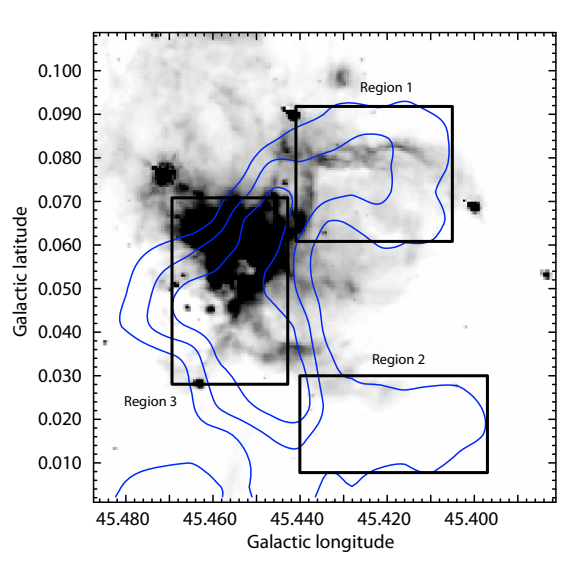

Region 1

Region 2
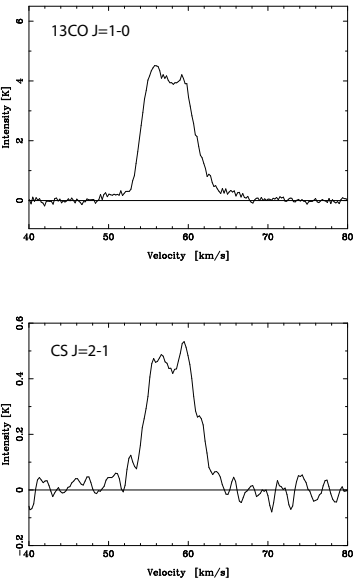
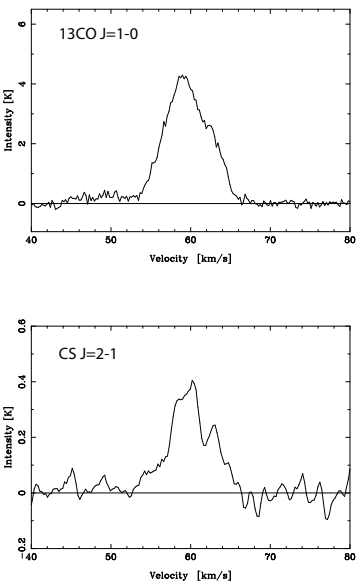

Region 3
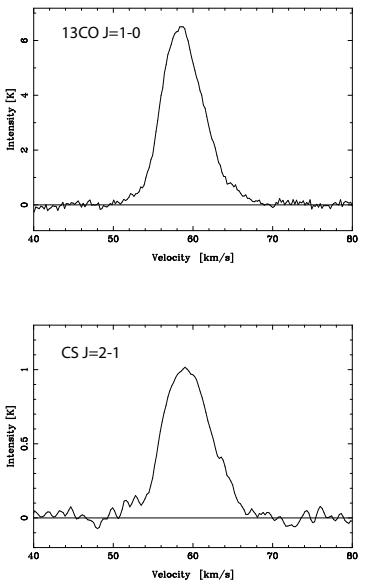

Fig. 6. Left: Spitzer-IRAC $8 \mu \mathrm{m}$ emission with contours of the integrated ${ }^{13} \mathrm{CO} J=1-0$ as presented in Fig. 1 . The boxes represent the regions from which were obtained the average of the molecular emissions. Towards the right the ${ }^{13} \mathrm{CO} J=1-0$ and CS $J=2-1$ average spectra from each region are shown. The rms noise are $\sim 0.03$ and $\sim 0.04 \mathrm{~K}$ for the ${ }^{13} \mathrm{CO}$ and $\mathrm{CS}$, respectively.

Table 2. Main parameters of the infrared sources found towards G45L.

\begin{tabular}{ccccccccccc}
\hline \hline$\#$ & Glimpse designation & Galactic coordinates & $J$ & $H$ & $K_{\mathrm{S}}$ & $3.6 \mu \mathrm{m}$ & $4.5 \mu \mathrm{m}$ & $5.8 \mu \mathrm{m}$ & $8.0 \mu \mathrm{m}$ & Notes \\
\hline 1 & G045.4260+00.0590 & $45^{\circ} .426,0 \circ 058$ & 13.93 & 11.89 & 11.08 & 10.47 & 10.55 & 10.21 & 10.18 & class III - (O-type) \\
2 & G045.4061+00.0506 & $45^{\circ} .406,0.050$ & 13.13 & 11.67 & 11.08 & 10.71 & 10.79 & 10.58 & 11.32 & \\
3 & G045.4255+00.0282 & $45^{\circ} .425,0.028$ & null & null & null & 13.7 & 12.3 & 11.09 & 10.05 & \\
4 & G045.4135+00.0528 & $45^{\circ} .413,0.052$ & 11.51 & 10.45 & 10.09 & 9.86 & 9.8 & 9.69 & 9.68 & class III \\
5 & G045.4250+00.0668 & $45^{\circ} .425,0.066$ & null & null & 14.43 & 11.33 & 10.31 & 9.77 & 9.81 & \\
6 & G045.4420+00.0415 & $45^{\circ} .442,0.041$ & null & null & null & 12.07 & 12.03 & 11.64 & 11.06 & \\
7 & G045.4437+00.0458 & $45^{\circ} .443,0.045$ & null & null & 12.67 & 10.68 & 10.36 & 9.81 & 9.84 & class III \\
8 & G045.4048+00.0438 & $45^{\circ} .404,0.043$ & null & 13.65 & 12.17 & 11.04 & 10.99 & 10.58 & 10.6 & class III - (O-type) \\
9 & G045.4274+00.0672 & $45^{\circ} .427,0 \circ 067$ & 13.11 & 11.64 & 11.07 & 10.53 & 10.49 & 9.94 & 9.63 & \\
10 & G045.4347+00.0627 & $45^{\circ} .434,0.062$ & null & null & null & 12.77 & 12.54 & 9.40 & 7.81 & class III \\
11 & G045.3969+00.0433 & $45^{\circ} .396,0.043$ & 11.79 & 10.41 & 9.85 & 9.55 & 9.6 & 9.41 & 9.39 & class III \\
12 & G045.4024+00.0412 & $45^{\circ} .402,0.041$ & 10.41 & 9.21 & 8.73 & 8.39 & 8.51 & 8.34 & 8.22 & class III \\
13 & G045.3976+00.0473 & $45^{\circ} .397,0.047$ & null & 13.67 & 12.42 & 11.5 & 11.33 & 10.82 & 10.89 & class III - (O-type) \\
14 & G045.4020+00.0361 & $45^{\circ} .402,0.036$ & 13.42 & 12.28 & 11.88 & 11.52 & 11.55 & 11.38 & 11.73 & clas \\
\hline
\end{tabular}

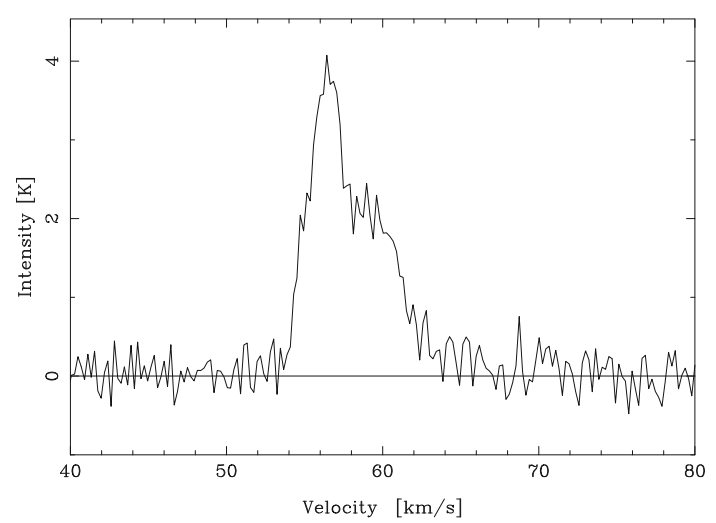

Fig. 7. ${ }^{13} \mathrm{CO} J=1-0$ spectrum obtained towards the middle PDR, at $l=$ $45^{\circ} .430, b=0.055$. The main component velocity is $v \sim 56 \mathrm{~km} \mathrm{~s}^{-1}$, which coincides with one of the two molecular components of the north PDR.

the four Spitzer-IRAC bands, we found 14 sources towards the region located inside the observed PDRs. The main parameters of these sources are shown in Table 2. Figure 8 indicates the location of the sources with respect to the emission distribution at $8 \mu \mathrm{m}$ (red) and the radio continuum at $20 \mathrm{~cm}$ (green). To examine the evolutionary stage of these sources, we analyze their location on a color-color IRAC diagram as shown in Fig. 9. Following Allen et al. (2004) color criteria, we found that 8 sources could be classified as main sequence stars (class III). Among these sources we look for O-type stars as responsible for ionizing the surrounding gas. Using the $J, H$ and $K$ apparent magnitudes as obtained from the 2MASS Point Source Catalog, we estimate the absolute magnitudes for the mentioned 8 sources. To convert the apparent magnitudes into absolute ones, we assume a distance of $8 \mathrm{kpc}$ and a visual absorption between 8 and 12 mag. The extinction values were obtained by inspecting the infrared source location in the color-color diagram $(H-K)$ vs. $(J-H)$ (not presented here). By comparing the estimated absolute magnitudes with those tabulated by Martins \& Plez (2006), we found that sources \#1, \#9 and \#14 are probably O-type stars (likely between $\mathrm{O} 4 \mathrm{~V}$ and $\mathrm{O} 8 \mathrm{~V}$ ), which is consistent with their position in the color-magnitude diagram $K$ vs. $(H-K$ ) (not presented here). Source \#1 is slightly displaced towards the region of giants stars in the color-magnitude diagram, but it could be a reddened O-type star. An inspection of Fig. 8 shows that sources \#1 and \#9 are located almost in the central part of G45L, while source \#14 lies towards its boundary. This fact suggest that sources \#1 and \#9 are the most promising candidates for being related to $\mathrm{G} 45 \mathrm{~L}$. In particular, source \#9 is projected onto a 


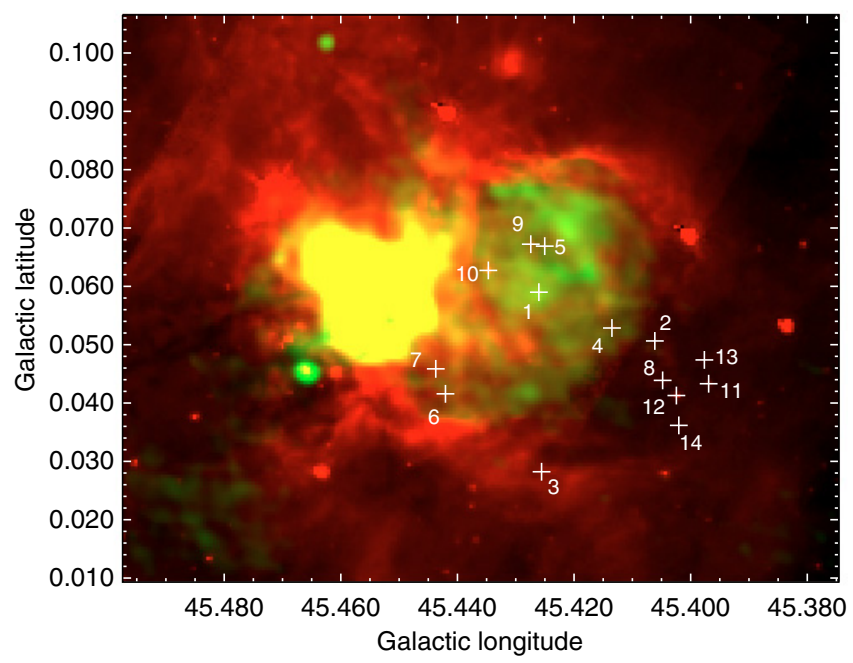

Fig. 8. Colour composite image, with the radio continuum emission at $1420 \mathrm{MHz}$ in green, and the infrared emission at $8 \mu \mathrm{m}$ in red. Yellow is the superposition of the IR and radio continuum emissions. The exciting star candidates are identified (see Table 2).

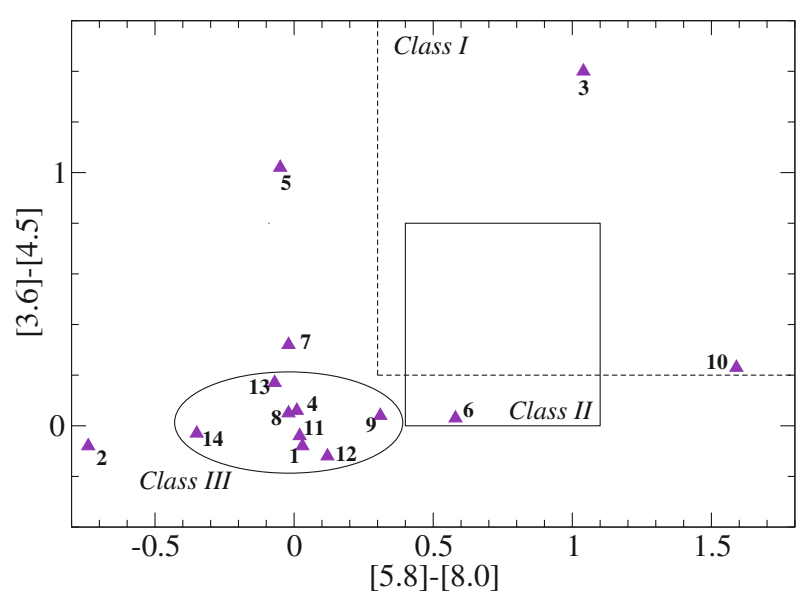

Fig. 9. GLIMPSE-IRAC color-color diagram [3.6]-[4.5] versus [5.8]-[8.0] for the sources displayed in Fig. 8. Class I-III regions are indicated following Allen et al. (2004). The ellipse (class III region: main sequence and giant stars) encloses the sources that we consider to be possible exciting star(s) of G45L.

local minimum observed in the brightest part of the G45L radio continuum emission, suggesting that this source could have been blowing into its environs creating a small cavity around itself.

In order to investigate whether these three sources can provide the energy necessary to ionize the gas, we need to estimate the radio continuum flux of $\mathrm{G} 45 \mathrm{~L}$. Using the radio continuum emission data at $1420 \mathrm{MHz}$, we estimate a flux density of $S_{1420 \mathrm{MHz}} \sim 1.0 \pm 0.2 \mathrm{Jy}$ for G45L. The number of UV photons necessary to keep the gas ionized is derived using $N_{\mathrm{UV}}\left(\right.$ photons s${ }^{-1}$ ) $=0.76 \times 10^{47} T_{4}^{-0.45} v_{\mathrm{GHz}}^{0.1} S_{v} D_{\mathrm{kpc}}^{2}$ (Chaisson 1976), where $T_{4}$ is the electron temperature in units of $10^{4} \mathrm{~K}$, $D_{\mathrm{kpc}}$ is the distance in kpc and $S_{v}$ is the flux density in Jy. Adopting an electron temperature of $10^{4} \mathrm{~K}$, we obtain $N_{\mathrm{UV}}=$ $(5 \pm 2) \times 10^{48} \mathrm{~s}^{-1}$. On the other hand, the UV photon flux corresponding to the contribution of the three stars is $\sim 3.3 \times 10^{49}$ photons per second (Martins et al. 2005). Thus, we conclude that the three stars can maintain the HII region G45L ionized and heat the dust emitting at $24 \mu \mathrm{m}$ in the ionized gas. It is important to mention that one of the central sources by itself, \#1 or \#9, can provide the necessary UV photons. On the contrary, source \#14 can not have created the HII region G45L alone.

Assuming that sources \#1, \#9, and \#14 are the sources responsible for the HII region G45L, we estimate its dynamical age using a simple model described by Dyson \& Williams (1980). In this model, the radius of the HII region at a given time $t$ is given by $R(t)=R_{\mathrm{S}}\left(1+7 c_{\mathrm{S}} t / 4 R_{\mathrm{S}}\right)^{4 / 7}$, where $c_{\mathrm{S}}$ is the sound velocity in the ionized gas $\left(c_{\mathrm{S}}=15 \mathrm{~km} \mathrm{~s}^{-1}\right)$ and $R_{\mathrm{s}}$ is the radius of the Strömgren sphere, given by $R_{\mathrm{S}}=\left(3 S_{*} / 4 \pi n_{\mathrm{o}}^{2} \alpha_{B}\right)^{1 / 3}$, where $\alpha_{B}=2.6 \times 10^{-13} \mathrm{~cm}^{3} \mathrm{~s}^{-1}$ is the hydrogen recombination coefficient at all levels above the ground level. $S_{*}$ is the total number of ionizing photons per unit of time emitted by the stars, and $n_{\mathrm{o}}$ is the original ambient density. As a rough estimate, $n_{\mathrm{o}}$ can be obtained by distributing the total molecular mass related to the structure $\left(M \sim 10^{5} M_{\odot}\right)$ over a sphere of about $7 \mathrm{pc}\left(3^{\prime}\right.$ at $\left.8 \mathrm{kpc}\right)$ in diameter, which yields $n_{0} \sim 10^{4} \mathrm{~cm}^{-3}$. Given that the actual diameter of G45L is about $7 \mathrm{pc}$, we infer that the HII region has been expanding for about $2 \times 10^{6} \mathrm{yr}$.

\subsection{Star formation around $G 45 L$}

To look for primary tracers of star formation activity around G45L, we use the GLIMPSE I Spring'07 Catalog to perform photometry. Considering only sources that have been detected in the four Spitzer-IRAC bands, we found 151 sources in the area delimited by the dashed circle shown in Fig. 10. The circular area was chosen in order to cover the molecular gas that surrounds the HII region and where the YSO candidates are expected to be located. This figure displays the spatial distribution of the YSO candidates over the Spitzer-IRAC $8 \mu \mathrm{m}$ emission. The green crosses indicate class I sources, the red boxes indicate class II or intermediate class I/II sources and the cyan circles indicate the sources that could be reddened class II objects. The source classification was performed according the photometric study presented in Fig. 11, which shows the IRAC color-color diagram of the sources found. The different regions indicated correspond to different stellar evolutionary stages, as defined by Allen et al. (2004). Seventeen sources lie in the region of the protostars with circumstellar envelopes (class I, green triangles), only 4 sources lie in the region of young stars with only disk emission (class II and intermediate class I/II, red triangles) and 104 sources lie in the region of the main sequence and giant stars (class III, blue triangles). Sources represented as cyan triangles, located outside the delimited regions, could therefore be reddened class II objects (Allen et al. 2004). Unfortunately we cannot perform an additional NIR photometric study because the 2MASS data from most of these sources are either missing or given as lower limits.

In Fig. 12 the same sources shown in Fig. 10 are displayed over the ${ }^{13} \mathrm{CO} J=1-0$ (left) and CS $J=2-1$ (right) integrated emissions between 54 and $66 \mathrm{~km} \mathrm{~s}^{-1}$. Of course we do not know if all the sources seen towards $\mathrm{G} 45 \mathrm{~L}$ are at the same distance as the HII region. However, the location of some of them, mainly the class I objects (green crosses in Fig. 12), suggest that they are possibly embedded in the molecular gas related to G45L. The class I objects lie preferently in the molecular shell and concentrate towards the east, where the CS $J=2-1$ emission peaks. They lie in the region where the EGO G45.47+0.05 is embedded (Cyganowski et al. 2008), confirming that in this area, southeast of the UCHII complex and eastern G45L, star formation is taking place.

As mentioned in Sect. 1, Feldt et al. (1998) and Blum \& McGregor (2008) identified several massive O-type stars that are 


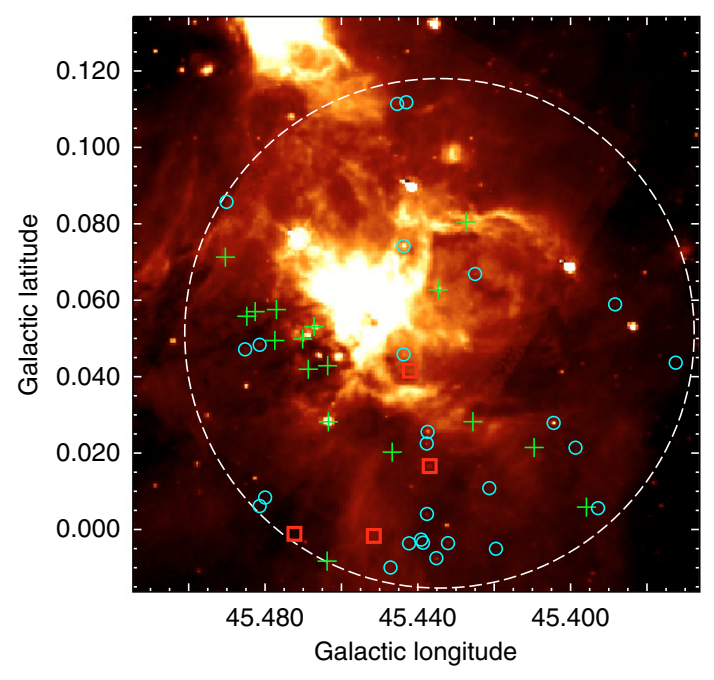

Fig. 10. Spitzer-IRAC $8 \mu \mathrm{m}$ emission with YSO candidates superimposed. Green crosses indicate class I sources, red boxes are class II or intermediate class I/II sources and the cyan circles are the sources that could be reddened class II objects. The circle encloses the region where the photometric study was performed.

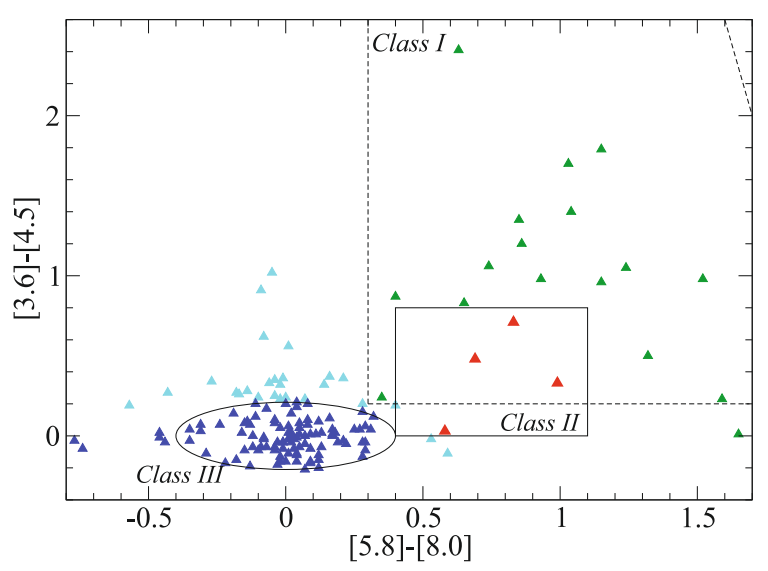

Fig. 11. GLIMPSE-IRAC color-color diagram [3.6]-[4.5] versus [5.8]-[8.0] for the sources observed inside the dashed circle displayed in Fig. 10. As in Fig. 9, class I-III regions are indicated following Allen et al. (2004). In this case we consider class I (green), class II and intermediate class I/II (red) and reddened class II (cyan) objects to study star formation around $\mathrm{G} 45 \mathrm{~L}$.

presumably on, or near, the zero-age main sequence, and are responsible for the ionization of the UCHII region G45.45+0.06. The authors also proposed that this UCHII region triggered the formation of younger UCHII regions in its surroundings, generating an UCHII complex. These studies do not mention any agent responsible for the formation of such stars. Taking into account the estimated age of G45L (about $2 \times 10^{6} \mathrm{yr}$ ), that star formation is taking place around it, probably through the collect and collapse process, and that the UCHII complex lies on one of its border, we propose that G45L could have triggered the formation of the zero-age main sequence stars that are ionizing G45.45+0.06. However we can not discard the possibility that both HII regions, G45.45+0.06 and G45L, could be coeval.

\subsection{HII region G45L spatial structure}

In this section, based on the IR emission and the molecular environment study, we attempt to describe the possible spatial structure of G45L.
As described in previous sections, a view of a larger area in the vicinity of the UCHII region G45.45+0.06 reveals that it actually lies on the border of a larger and fainter HII region that we called G45L. The morphological study of G45L suggests that this HII region is far from being spherical. The observed morphology is quite complex and projection effects should be considered to analyze the three dimensional structure. In this way, taking into account the location of the PDRs together with the spatial and velocity molecular gas distribution, we suggest that G45L has a pear shape, as is sketched in Fig. 13. This figure presents two sketches of G45L possible structure as seen from two different positions. Figure 13 (left) reveals that the north PDR is composed of two structures seen superimposed along the line of sight and the middle PDR is the projection of the southern border of the HII region portion closer to us. The UCHII complex that lies to the east of G45L is represented as a red cloud. Figure 13 (right) shows a longitudinal cut of G45L indicating the molecular gas components related to the PDRs. As the molecular analysis shows, the structure delimited by the north and the middle PDRs has associated molecular gas centered at $v \sim 55 \mathrm{~km} \mathrm{~s}^{-1}$, while the structure delimited by the south and the north PDRs has associated molecular gas centered at $v \sim 59 \mathrm{~km} \mathrm{~s}^{-1}$.

Most ionized nebulae, in particular HII regions, have complex morphologies (Morisset et al. 2005). There are many physical causes that may account for such morphologies, such as the presence of density gradients in the ISM where the HII region is evolving, the existence of more than one exciting star, the fact that these exciting stars could have high spatial velocities with respect to the local ISM, and the effects of possible stellar and/or interstellar magnetic fields.

\section{Summary}

Using multiwavelength survey and archival data, we studied the ISM towards a region of about $7^{\prime} \times 7^{\prime}$ in the vicinity of the G45.45+0.06 UCHII complex. The main results can be summarized as follows:

(a) We found that the UCHII complex lies on the border of a larger $\left(\sim 3^{\prime}\right.$ of diameter $)$ and fainter HII region, here named G45L.

(b) Although G45L is not completely bordered by a PDR, its morphology resembles the structure of the IR dust bubbles associated with $\mathrm{O}$ and early-B type stars: a PDR visible in the $8 \mu \mathrm{m}$ band, which encloses ionized gas observed at $20 \mathrm{~cm}$ and hot dust observed at $24 \mu \mathrm{m}$.

(c) We find a good morphological correlation between the PDRs and the molecular gas, which suggests that the HII region may be collecting the molecular material.

(d) Taking into account the velocity $\left(v_{\mathrm{LSR}} \sim 55-60 \mathrm{~km} \mathrm{~s}^{-1}\right)$ of the molecular gas related to G45L and the HI absorption study, we conclude that this HII region is at the same distance as the UCHII complex, $\sim 8 \mathrm{kpc}$.

(e) The PDR position and the spatial and velocity distribution of the associated molecular shell suggest that G45L has a pear shape.

(f) From a near- and mid-IR photometric study, we found three sources, likely O-type stars (between O4V and O8V) that are possibly responsible for the creation of G45L. Additionally, we found several YSO candidates lying preferently in the molecular shell and concentrated towards the east where the CS $J=2-1$ emission peaks. Our results confirm that the southeastern region of the UCHII complex 

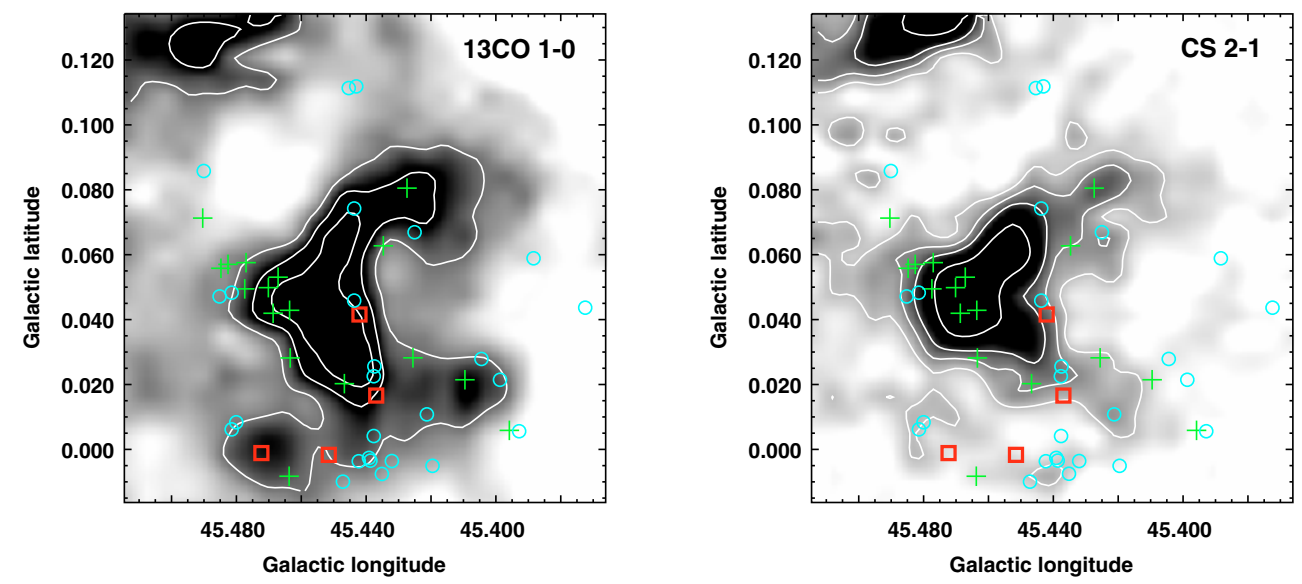

Fig. 12. Left: ${ }^{13} \mathrm{CO} J=1-0$ integrated emission between 54 and $66 \mathrm{~km} \mathrm{~s}^{-1}$ with YSO candidates superimposed. The contour levels are 27,37 and $48 \mathrm{~K} \mathrm{~km} \mathrm{~s}^{-1}$. Right: same, but grays are the CS $J=2-1$ integrated emission in the same velocity range. The contour levels are $2.4,3.6,5$ and $8 \mathrm{~K} \mathrm{~km} \mathrm{~s}^{-1}$. Green crosses indicate class I sources, red boxes are class II or intermediate class I/II sources and the cyan circles are the sources that could be reddened class II objects.
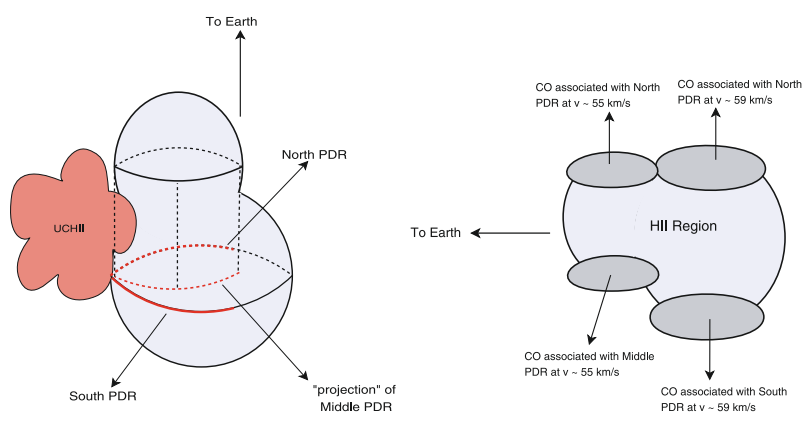

Fig. 13. Left: sketch of the possible shape of the HII region G45L. The PDRs are marked. The UCHII complex is represented as a red cloud. Right: a longitudinal cut of G45L showing the molecular gas components related to the PDRs.

where G45.45+0.06 is embedded (eastern part of G45L) is an active star formation region.

(g) Assuming that three O-type stars are responsible for G45L, we suggest this HII region has been expanding for about $2 \times$ $10^{6} \mathrm{yr}$ and could have triggered the formation of the zeroage main sequence stars that are ionizing the UCHII region G45.45+0.06. However we cannot discard that both HII regions are coeval.

Acknowledgements. We wish to thank the anonymous referee whose comments and suggestions have helped to improve the paper. S.P. and S.C. are members of the Carrera del investigador cientifico of CONICET, Argentina. M.O. is a doctoral fellow of CONICET, Argentina. This work was partially supported by the CONICET grant PIP 112-200801-02166, UBACYT A023 and ANPCYT PICT2007-00902 and -00812.

\section{References}

Allen, L. E., Calvet, N., D’Alessio, P., et al. 2004, ApJS, 154, 363 Anderson, L. D., Bania, T. M., Jackson, J. M., et al. 2009, ApJS, 181, 255 Blum, R. D., \& McGregor, P. J. 2008, AJ, 135, 1708
Chaisson, E. J. 1976, in Frontiers of Astrophysics, 259

Churchwell, E., Povich, M. S., Allen, D., et al. 2006, ApJ, 649, 759 Churchwell, E., Watson, D. F., Povich, M. S., et al. 2007, ApJ, 670, 428

Cichowolski, S., Romero, G. A., Ortega, M. E., Cappa, C. E., \& Vasquez, J. 2009, MNRAS, 394, 900

Codella, C., Lorenzani, A., Gallego, A. T., Cesaroni, R., \& Moscadelli, L. 2004, A\&A, 417, 615

Cyganowski, C. J., Whitney, B. A., Holden, E., et al. 2008, AJ, 136, 2391

Deharveng, L., Zavagno, A., \& Caplan, J. 2005, A\&A, 433, 565

Deharveng, L., Lefloch, B., Kurtz, S., et al. 2008, A\&A, 482, 585

Dyson, J. E., \& Williams, D. A. 1980, Physics of the interstellar medium (New York, Halsted Press)

Fazio, G. G., Hora, J. L., Allen, L. E., et al. 2004, ApJS, 154, 10

Feldt, M., Stecklum, B., Henning, T., et al. 1998, A\&A, 339, 759

Garay, G., Rodriguez, L. F., Moran, J. M., \& Churchwell, E. 1993, ApJ, 418, 368

Giveon, U., Becker, R. H., Helfand, D. J., \& White, R. L. 2005a, AJ, 129, 348

Giveon, U., Becker, R. H., Helfand, D. J., \& White, R. L. 2005b, AJ, 130, 156

Goicoechea, J. R., Pety, J., Gerin, M., et al. 2006, A\&A, 456, 565

Hatchell, J., Thompson, M. A., Millar, T. J., \& MacDonald, G. H. 1998, A\&AS, 133,29

Johnstone, D., Boonman, A. M. S., \& van Dishoeck, E. F. 2003, A\&A, 412, 157

Jackson, J. M., Rathborne, J. M., Shah, R. Y., et al. 2006, ApJS, 163, 145

Kolpak, M. A., Jackson, J. M., Bania, T. M., Clemens, D. P., \& Dickey, J. M. 2003, ApJ, 582, 756

Luna, A., Bronfman, L., Carrasco, L., \& May, J. 2006, ApJ, 641, 938

Martins, F., \& Plez, B. 2006, A\&A, 457, 637

Martins, F., Schaerer, D., \& Hillier, D. J. 2005, A\&A, 436, 1049

Matthews, H. E., Goss, W. M., Winnberg, A., \& Habing, H. J. 1977, A\&A, 61, 261

Morisset, C., Stasińska, G., \& Peña, M. 2005, MNRAS, 360, 499

Ohashi, N., Kawabe, R., Ishiguro, M., \& Hayashi, M. 1991, AJ, 102, 2054

Pomarès, M., Zavagno, A., Deharveng, L., et al. 2009, A\&A, 494, 987

Remijan, A., Shiao, Y.-S., Friedel, D. N., Meier, D. S., \& Snyder, L. E. 2004, ApJ, 617, 384

Simon, R., Jackson, J. M., Clemens, D. P., Bania, T. M., \& Heyer, M. H. 2001, ApJ, 551, 747

Stil, J. M., Taylor, A. R., Dickey, J. M., et al. 2006, AJ, 132, 1158

Watson, C., Povich, M. S., Churchwell, E. B., et al. 2008, ApJ, 681, 1341

Werner, M. W., Roellig, T. L., Low, F. J., et al. 2004, ApJS, 154, 1

White, R. L., Becker, R. H., \& Helfand, D. J. 2005, AJ, 130, 586

Wilner, D. J., Ho, P. T. P., \& Zhang, Q. 1996, ApJ, 462, 339

Wood, D. O. S., Churchwell, E., \& Salter, C. J. 1988, ApJ, 325, 694

Zavagno, A., Pomarès, M., Deharveng, L., et al. 2007, A\&A, 472, 835 